\title{
Complicaciones vasculares asociadas a la enfermedad inflamatoria intestinal
}

\author{
R. BAÑOS MADRID, H. SALAMA BENARROCH, J. MARTÍNEZ CRESPO, \\ S. MORÁN SÁNCHEZ, A. VARGAS ACOSTA, J. MERCADER MARTÍNEZ \\ Servicio de Medicina del Aparato Digestivo. Hospital Universitario Virgen de la Arrixaca \\ Murcia
}

\author{
VASCULAR COMPLICATIONS ASSOCIATED WITH INFLAMMA - \\ TORY BOWEL DISEASE
}

\begin{abstract}
RESUMEN
Los fenómenos tromboembólicos son una manifestación extraintestinal bien conocida de la enfermedad inflamatoria intestinal, pero rara en la practica clínica. Estudios histológicos y hematológicos sugieren que el síndrome de hipercoagulabilidad presente en esta enfermedad está involucrado en la patogénesis de la enfermedad inflamatoria intestinal. De todas maneras el mecanismo exacto de hipercoagulabilidad aún no es bien conocido. Durante la fase de actividad de la enfermedad hay un aumento de los niveles de factor VIII, fibrinógeno, plaquetas, Factor V y un descenso en los niveles de antitrombina III. Hay una correlación clara entre estas alteraciones hematológicas y la actividad de la enfermedad. Presentamos dos pacientes con enfermedad inflamatoria intestinal y síndrome de hipercoagulabilidad. Se revisa la literatura y se comentan los mecanismos patogénicos.
\end{abstract}

PALABRAS CLAVE: Enfermedad inflamatoria intestinal. Manifestaciones extraintestinales. Hipercoagulabilidad.

\begin{abstract}
Thromboembolic episode is a well known extraintestinal manifesta tion of inflammatory bowel disease, but it is a clinical rare complication. Histological and hematological studies suggest that a hypercoagulable state is involved in the pathogenesis of inflammatory bowel disease. However, the exact mechanism of hypercoagulability is still unknown. During the acute recurrences there is an increase of factor VIII, fibrino gen, platelet, factor $V$ and decrease of antithrombin III. Hematologic disorders seem markedly correlated with the activity of the disease. We report on two patients with Inflammatory bowel disease and hypercoa gulable state. We review the literature and discuss about the pathogenic mechanisms of such complication.
\end{abstract}

KEY WORDS: Inflammatory bowel disease. Extraintestinal manifesta tions. Hypercoagulability.

Baños Madrid R, Salama Benarroch H, Martínez Crespo J, Morán Sánchez S, Vargas Acosta A, Mercader Martínez J. Complicaciones vasculares asociadas a la enfermedad inflamatoria intestinal. An Med Interna (Madrid) 2003; 20: 81-84.

\section{INTRODUCCIÓN}

La Enfermedad Inflamatoria Intestinal (EII) se comporta como una enfermedad sistémica, implicando a diferentes órganos y sistemas extraintestinales (1). Los fenómenos tromboembólicos son una complicación relativamente frecuente de la EII, resultado de la hipercoagulabilidad asociada a dicha enfermedad. La incidencia de tromboembolismo varía de un $1,2 \%-7,5 \%$ en los estudios clínicos de pacientes vivos, a un $6,6 \%-39 \%$ en los estudios necrópsicos $(2,3)$. Las trombosis pueden ser venosas o arteriales, siendo la localización más frecuente a nivel del sistema venoso profundo de las extremidades inferiores $(84,2 \%)$ (4), pudiendo producirse secundariamente tromboembolismo de pulmón. El riesgo de complica- ciones tromboembólicas es mayor durante la fase activa de la enfermedad intestinal y añaden un mal pronóstico a la evolución.

Las alteraciones de la coagulación encontradas en los pacientes con EII activa incluyen trombocitosis, elevación del fibrinógeno y factores V, VIII, descenso de los niveles de antitrombina III y alteraciones de la fibrinolisis. En la mayoría de los pacientes, éste estado de hipercoagulabilidad no existe cuando la EII está inactiva (5).

Presentamos dos casos de pacientes con diagnostico de enfermedad inflamatoria intestinal y complicaciones vasculares en el curso de su enfermedad, con la peculiaridad de que en uno de dichos casos coexistió la trombosis arterial y la venosa en la misma paciente.

Trabajo aceptado: 19 de noviembre de 2001

Correspondencia: Ramón Baños Madrid. C/ Alameda de San Antón. n 38- 1 B. 30205 Cartagena. Murcia. 


\section{CASOS APORTADOS}

Caso 1: Mujer de 27 años diagnosticada de enfermedad de Crohn a los 21 años, con afectación perianal y artritis de grandes articulaciones. A los 3 años de evolución presentó episodio de isquemia arterial aguda en ambas extremidades mas acusada en la derecha, confirmándose en la arteriografía obstrucción de la arteria poplítea derecha y tronco tibioperoneo derecho, con revascularización de la tibial posterior a $3 \mathrm{~cm}$, y de la peronea a $10 \mathrm{~cm}$. Estos hallazgos fueron similares en la extremidad inferior izquierda (Fig. 1). Tratada con heparina sódica a dosis de anticoagulación la evolución posterior fue buena. Fue dada de alta con tratamiento antiagregante, salazopyrina y corticoides a dosis de mantenimiento.

Reingresa dos años después por clínica de dolor y edema en pierna derecha con gran afectación del estado general. La exploración cardiorrespiratoria y abdominal era normal. La pierna derecha estaba edematosa y con aumento de la temperatura a ese nivel. La paciente presentaba intenso dolor a la palpación en la pantorrilla derecha y gran impotencia funcional siendo el pulso pedio derecho débil. Analíticamente destacaba una leucocitosis de 16.000 con desviación izquierda, Hgb: 8 gr/dl, Hto: 28\%, plaquetas: 600.000,Vsg: $45 \mathrm{~mm} /$ $1^{\circ}$ hora, fibrinógeno: $345 \mathrm{mg}$, quick: $60 \%$. Proteínas, albúmina, hierro y calcio bajos. Radiografía de tórax y abdomen sin alteraciones significativas.

Una ecodoppler de la extremidad inferior derecha confirma la presencia de una trombosis al nivel de la vena femoral profunda dcha. Se trata con anticoagulación con heparina sódica, durante 10

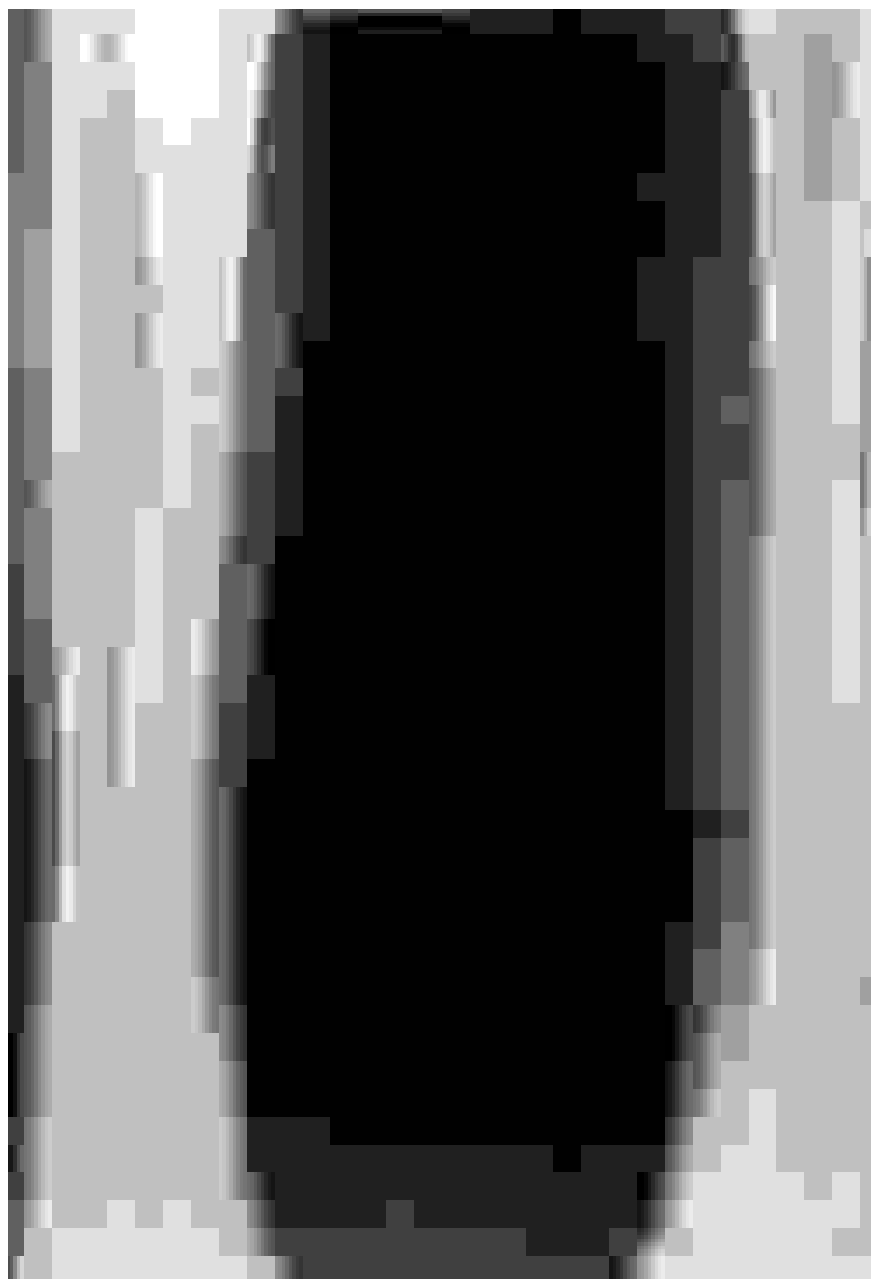

Fig. 1. La arteriografía muestra la obstrucción de la arteria poplítea y tronco tibioperoneo de ambas extremidades. días, introduciéndose dicumarínicos a partir del séptimo día, manteniendo un INR entre 2-3.

Coincidiendo con este ingreso se realiza estudio de hipercoagulabilidad, los factores V y VIII son del 97 y del 88\%, respectivamente, la actividad de la antitrombina III, proteína $\mathrm{S}$ y plasminógeno fueron normales. La proteína $\mathrm{C}$ funcional fue del $49 \%$ y la antigénica del $60 \%$, presentando anticuerpos antifosfolípidos positivos. La paciente era portadora de una deficiencia heterocigótica tipo 1 de la proteína $C$ y de un síndrome antifosfolípido.

Dada de alta con tratamiento ininterrumpido con dicumarínicos no ha presentado ningún otro proceso trombótico.

Caso 2: Mujer de 18 años de edad que consulta por diarrea con $5 / 6$ deposiciones al día con sangre, moco, pus y fiebre de hasta $39^{\circ} \mathrm{C}$ de 10 días de evolución. Entre los antecedentes personales destaca el diagnostico de colitis ulcerosa un año antes y que estaba sin tratamiento en el momento del ingreso. A la exploración presentaba palidez marcada, fiebre de $38^{\circ} \mathrm{C}$ y signos de malnutrición. Auscultación cardíaca rítmica, con taquicardia a $125 \mathrm{lpm}$ y no presentaba soplos. Auscultación pulmonar normal. Abdomen no doloroso sin megalias. Extremidades, articulaciones y piel normales. Tacto rectal con sangre en ampolla rectal.

Analíticamente destacaba una leucocitosis de 14.500 con desviación izquierda, anemia severa (Hgb-8gr/dl, Hto-28\%), plaquetas de 454.000, Vsg de $50 \mathrm{~mm} / 1^{\mathrm{a}}$ hora. Fibrinógeno: $530 \mathrm{mg}$. Quick: $48 \%$. Radiografía de tórax y abdomen sin hallazgos significativos. La colonoscopia mostró una colitis ulcerosa típica con afectación severa hasta ángulo esplénico.

A su ingreso se inicia tratamiento con corticoides intravenosos y 5-ASA, con mala respuesta.

En el decimocuarto día de ingreso presenta una hemianopsia izquierda brusca, desarrollando posteriormente una amaurosis bilateral total y varios episodios de convulsiones tónico-clónicas generalizadas, que cedieron con fenitoína. En la exploración neurológica destacaba una amaurosis bilateral con fondo de ojo normal. Se realizó un electroencefalograma en el que aparecía un sufrimiento córtico-subcortical en áreas posteriores de ambos hemisferios. En la TAC craneal se evidenciaba una lesión hipodensa en región occipital derecha (Fig. 2). La ecodoppler carotídea resultó normal. Se realiza estudio de hipercoagulabilidad destacando un factor $\mathrm{V}$ : $151 \%$, factor VIII: $148 \%$ y un fibrinógeno de $515 \mathrm{mg}$, resto normal. La paciente presentaba una elevación moderada de dichos factores.

Con el diagnóstico de ACV isquémico occipital derecho se inicia tratamiento con heparina sódica y antiagregación con mejoría progresiva de su cuadro neurológico. La paciente es dada de alta con tratamiento con dicumarínicos de forma permanente.

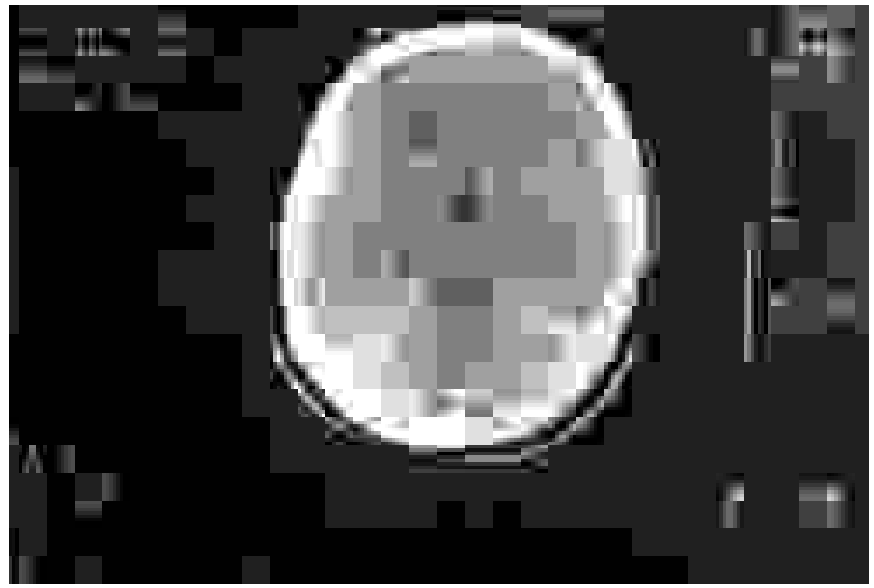

Fig. 2. TAC craneal donde se aprecia una imagen hipodensa en región occipital derecha, compatible con un ACV isquémico. 


\section{DISCUSIÓN}

El síndrome de hipercoagulabilidad asociado a la enfermedad inflamatoria intestinal fue descrito por primera vez por Bargen y Barker en 1936 (6). La EII se acompaña de numerosas manifestaciones extraintestinales, entre ellas un estado de hipercoagulabilidad que puede dar lugar a fenómenos tromboembólicos, vasculitis e incluso isquemias arteriales. La afectación venosa es más frecuente que la arterial y se han descrito diversas localizaciones, siendo las más frecuentes la afectación de las arterias y venas ileofemorales, pulmonares y cerebrales (7).

Clásicamente se ha considerado a la EII como un estado de hipercoagulabilidad secundario (Tabla I), estando afectada tanto la hemostasia primaria como la coagulación y la fibrinolisis. Las plaquetas aumentan en número, como reactante de fase aguda, habiéndose demostrado también una mayor agregabilidad y recambio plaquetario aún en fases inactivas de EII (8). Las alteraciones de la coagulación son fundamentalmente cuantitativas, con aumento de los factores I, V, VIII antígeno (factor más frecuente e importante y que está en relación con la actividad y la extensión), déficit de antitrombina III y cualitativas, con formación de sustancias procoagulantes dependientes del sistema mononuclear fagocítico, que puede activarse por endotoxinas de bacterias intestinales, mecanismos inmunológicos celulares y perdida de proteoglucanos del endotelio. La antitrombina III es un inhibidor de la coagulación, y su deficiencia sola, o más probablemente asociada a las otras alteraciones, puede predisponer a complicaciones vasculares. El origen del descenso de niveles de antitrombina III no está completamente aclarado, postulándose que puede reflejar un incremento en su consumo o destrucción en la zona de inflamación y, en menor medida, una pérdida de proteínas a través del tubo digestivo.

Los anticuerpos antifosfolípido, anticardiolipina, también han sido descritos en asociación con la EII (7). La hipofibri-

\section{TABLA I}

MECANISM OS PRINCIPALES DE HIPERCO AGULABILIDAD SECUNDARIA ASO CIADOS A LA EII

Aumento de Plaquetas

Aumento de fibrinógeno

Aumento del Factor V, VIII, IX

Disminución antitrombina III

Disminución fibrinolisis

Encamamiento

Deshidratación nolisis es un factor que también se ha demostrado importante, sobre todo en pacientes con EII en actividad, esta alteración de la capacidad del sistema fibrinolítico podría contribuir a un riesgo aumentado de complicaciones tromboembólicas y a la patogénesis de la enfermedad inflamatoria intestinal (10).

La mayoría de las alteraciones mencionadas se ponen de manifiesto durante la fase activa de enfermedad, siendo rara la aparición de fenómenos vasculares sin brote intestinal (5). En algunas ocasiones la tendencia trombótica no dependería de la actividad inflamatoria, postulándose que fuera ésta secundaria a la primera (11). En 1986 Wakefield implicó a los fenómenos tromboembólicos microvasculares en la patogénesis de la enfermedad de Crohn $(12,13)$, siendo desde entonces esta teoría reafirmada por diferentes estudios experimentales, histológicos e incluso epidemiológicos, que demuestran una menor prevalencia de EII en pacientes con déficit hereditarios de factores de la coagulación (14-16). Algunos autores recomiendan el tratamiento con heparina de bajo peso molecular en pacientes con EII y en brote intestinal, aunque no tengan antecedentes tromboembólicos apoyándose en la teoría anterior $(17,18)$. En la actualidad se recomienda la aplicación profiláctica de heparina a todo paciente con brote intestinal, sobre todo si presenta otros factores de hipercoagulabilidad asociados, como el encamamiento prolongado o deshidratación.

El tratamiento con heparina no es motivo de controversia actualmente en los pacientes con complicaciones tromboembólicas, debiendo instaurarse cuanto antes para evitar nuevos eventos trombóticos. En aquellos pacientes con tendencia hereditaria a la trombosis se recomienda la anticoagulación oral durante un tiempo prolongado (19). La existencia de episodios de trombosis de repetición, junto a la resistencia para alcanzar un buen nivel de anticoagulación con la dosis de heparina convencional en un paciente con enfermedad inflamatoria intestinal, debe hacer sospechar la existencia de un factor adicional responsable del mencionado cuadro clínico, además del fenómeno de hipercoagulabilidad secundaria asociada a la EII. Consideramos que la investigación de un estado de trombofília primario (deficiencia de proteína $\mathrm{C}$, proteína $\mathrm{S}$, antitrombina III y mutación de Leiden del factor $\mathrm{V}$ ) es obligada aun en procesos que generan un estado de hipercoagulabilidad secundaria como es el caso de la EII $(20,21)$. La deficiencia cuantitativa o funcional de cualquiera de estas proteínas plasmáticas provoca tendencia a trombosis. La resistencia del Factor $\mathrm{V}$ a la degradación por la Proteína $\mathrm{C}$ activada se produce como consecuencia de una mutación del gen del Factor V, la proteína resultante se llama Factor V Leiden (22). Otra causa de trombofília primaria recientemente descubierta es la mutación del gen de la protrombina G20210A, su presencia junto al anterior podría jugar un importante papel en la patogénesis e incrementar el riesgo de complicaciones trombóticas en los pacientes con EII y portadores de dichas alteraciones (23).

\section{Bibliografía}

1. Danci JT. Extraintestinal manifestation of idiopathic inflammatory bowel disease. Arch Intern Med 1998; 148: 297-302.

2. Talbot RW, Heppell J, Dozois RR, Beart RW Jr. Vascular complications of inflammatory bowel disease. Mayo Clin Proc 1986; 61: 140-5.

3. Graef V, Baggenstoss AH, Saber WG. Venous thrombosis occurring in nonspecific ulcerative colitis. A necropsy study. Arch Intern Med 1966; 177: $377-82$
4. Novacek, Miehsler W, Kapiotis S, Katzenschlager R, Speiser W, Vogelsang H. Thromboembolism and resistance to activated protein C in patients with inflammatory bowel disease. Am J Gastroenterol 1999; 94 (3): 685-90.

5. López Morante AJ, Prieto García M, Yuguero del Moral F, Sáez-Royuela F, Martín Lorente JL, Ojeda Giménez C. Alteraciones de la coagulación en la enfermedad inflamatoria intestinal. Rev Clin Esp 1992; 190: 18-21. 
6. Bargen JA, Barker NW. Extensive arterial and venous thrombosis complicating chronic ulcerative colitis. Arch Intern Med 1936; 58: 17-31.

7. Hudson M, Chitolie A, Hutton RA, Smith MS, Pounder RE, Wakefield AJ. Trombotic vascular risk factor in inflammatory bowel disease. Gut 1996; 38: 733-7.

8. Alcalde Encinas MM, Pérez Gracia A, Hallal H, Bautista de Prado J, Conesa Pallarés FJ, Vilaplana García R. Trombosis de senos venosos cerebrales y colitis ulcerosa. Rev Esp Enferm Dig 2000; 92: 105-8.

9. Aadland E, Odegaard OR, Rseth A. Free protein S deficiency in patient with chronic inflamatory bowel disease. Scand J Gastroenterol 1992; 27: 957-60.

10. Suárez Crespo JF, Nogueras López F, de Teresa Galván FJ, de Sola Earle CM, González Galilea A, et al. Complicaciones tromboembólicas en la enfermedad inflamatoria intestinal. Gastroenterol Hepatol 1997; 20: 180-3.

11. Yerby MS, Bailey GM. Superior sagittal sinus thrombosis 10 years after surgery for ulcerative colitis. Stroke 1980; 11: 294-6.

12. Thompson NP, Wakefield AJ, Pounder RE. Inherited disorders of coagulation appear to protect against inflammatory bowel disease. Gastroenterology 1995; 108 (4): 1011-5.

13. Wakefield AJ, Sawyer AM, Dhillon AP, Pittilo RM, Rowles PM, Lewis AA, Poundez RE. Pathogenesis of Crohn's disease: multifocal gastrointestinal infarction. Lancet 1989; 2: 1057-62.

14. Pounder RE. The pathogenesis of Crohn's disease. J Gastroenterol 1994; 29: 11-5

15. Mose L, Sim R, Hudson M, Dhillon AP, Pounder R, Wakefield AJ. Inmunohistochemical study of tissue factor expresion in normal intestine and idiopathic inflammatory bowel disease. J Clin Pathol 1993; 46: 703-8.

16. Thompson NP, Wakefield AJ, Pounder RE. Inherited disorders of coagulation appear against inflammatory bowel disease. Gastroenterology 1995; 108: 1011-5.

17. Zaugodniy LE, Mustyats AP. The use of anticoagulants in combined therapy of non specific ulcerative colitis. Klin Med 1982; 60: 74-80.

18. Gaffney PR, Doyle CT, Gaffney A, Hogan J, Mayes DP, Annis P. Paradoxical response to heparin in 10 patients with ulcerative colitis. Am J Gastroenterol 1995; 90: 220-3.

19. Lake AM, Stauffer JQ, Stuart MJ. Hemostatic alterations in inflammatory bowel disease: response to therapy. Am J Dig Dis 1978; 23 (10): 897-902.

20. Hudson M, Hutton RA, Wakefield AJ, Sawyer AM, Pounder RE. Evidence for activation of coagulation in Crohn's disease. Blood Coagul Fibrinolysis 1992; 3: 773-8.

21. Zauber NP, Sabbath-Solitare M, Rajoria G, Mogan G. Factor V Leiden mutation is not increased in patients with inflammatory bowel disease. J Clin Gastroenterol 1998; 27 (3): 215-6.

22. Haslam N, Standen GR, Probert CS. An investigation of the association of the factor V Leiden mutation and inflammatory bowel disease. Eur J Gastroenterol Hepatol 1999; 11 (11): 1289-91.

23. Papa A, De Stefano V, Gasbarrini A, Chiusolo P, Cianci R, Casorel A, et al. Prevalence of factor V Leiden and the G20210A prothrombin -gen mutation in inflammatory bowel disease. Blood Coagul Fibrinolysis $2000 ; 11$ (5): 499-503. 